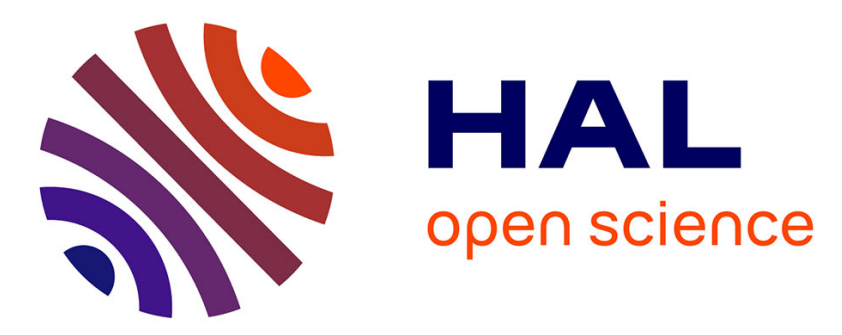

\title{
Polarized Parallel Transport and Uniruled Divisors on Deformations of Generalized Kummer Varieties
}

\author{
Giovanni Mongardi, Gianluca Pacienza
}

\section{To cite this version:}

Giovanni Mongardi, Gianluca Pacienza. Polarized Parallel Transport and Uniruled Divisors on Deformations of Generalized Kummer Varieties. International Mathematics Research Notices, 2018, 2018 (11), pp.3606-3620. 10.1093/imrn/rnw346 . hal-03256249

\section{HAL Id: hal-03256249 \\ https://hal.science/hal-03256249}

Submitted on 4 Jul 2021

HAL is a multi-disciplinary open access archive for the deposit and dissemination of scientific research documents, whether they are published or not. The documents may come from teaching and research institutions in France or abroad, or from public or private research centers.
L'archive ouverte pluridisciplinaire $\mathbf{H A L}$, est destinée au dépôt et à la diffusion de documents scientifiques de niveau recherche, publiés ou non, émanant des établissements d'enseignement et de recherche français ou étrangers, des laboratoires publics ou privés. 
G. Mongardi and G. Pacienza (2019) "Corrigendum and Addendum to "Polarized Parallel Transport and Uniruled Divisors on Generalized Kummer Varieties","

International Mathematics Research Notices, Vol. 00, No. 0, pp. 1-7

doi:10.1093/imrn/rnz163

\section{Corrigendum and Addendum to "Polarized Parallel Transport and Uniruled Divisors on Generalized Kummer Varieties"}

\section{Giovanni Mongardi, ${ }^{1, *}$ and Gianluca Pacienza ${ }^{2}$}

${ }^{1}$ Dipartimento di Matematica, Alma Mater Studiorum Universitá di Bologna, Piazza di Porta San Donato 5, Bologna 40126, Italia and ${ }^{2}$ Institut Elie Cartan de Lorraine, Université de Lorraine, BP 70239, Vandoeuvre-lés-Nancy Cedex F-54506, France

*Correspondence to be sent to: e-mail: giovanni.mongardi2@unibo.it

We correct the statement of the main result of [9] and provide some further precisions.

The goal of this short note is to state correctly the main result of [9]. For the definitions, the notations and the motivations we refer the reader to [9]. The correct statement is the following:

Theorem 0.1. Let $n \geq 1$ be an integer. Let $\mathfrak{M}=\cup_{d>0} \mathfrak{M}_{2 d}$ be the union of the moduli spaces $\mathfrak{M}_{2 d}$ of projective irreducible holomorphic symplectic varieties of $K_{n}(A)$-type polarized by a line bundle of degree $2 d$. For all $(X, H) \in \mathfrak{M}$, outside at most a finite number of connected components determined by the monodromy orbit of $H$, the linear system $|m H|$, for some $m$, contains a uniruled divisor covered by rational curves of primitive class.

Let $q$ be the Beauville-Bogomolov quadratic form on $H^{2}(X, \mathbb{Z})$. This induces an embedding $H^{2}(X, \mathbb{Z}) \hookrightarrow H_{2}(X, \mathbb{Z}), H \mapsto H^{\vee}$. By abuse of notation we denote again by $q$ the quadratic form on $H_{2}(X, \mathbb{Z})$. 
Remark 0.2. The statement above insures precisely existence of uniruled divisors covered by primitive rational curves if there exist integers $p, g$, and $\epsilon$ such that $p \geq g$ and $\epsilon=0$ or 1 with

(i) the class $\alpha:=\frac{H^{\vee}}{\operatorname{div}(H)} \in H_{2}(X, \mathbb{Z})$ can be written as $\gamma+(2 g-\epsilon) \eta$ with $\eta$ in the monodromy orbit of the class of the exceptional curve on a $K_{n}(A)$;

(ii) $\gamma \in \eta^{\perp}, q(\gamma)=2 p-2$ (hence, $\left.q(\alpha)=2 p-2-\frac{(2 g-\epsilon)^{2}}{2 n+2}\right)$.

\section{Remark 0.3.}

(i) It follows from Proposition 2.1 that if $q(\alpha)>n+1$, then a multiple of $H$ is uniruled by primitive rational curves of class $\alpha$.

(ii) If $\rho(X) \geq 2$ then $X$ always contains an ample uniruled divisor covered by primitive rational curves (cf. Corollary 2.3).

(iii) If $n \leq 5$ then the conclusion of the theorem holds for all the connected components of $\mathfrak{M}$ (cf. Remark 2.3).

(iv) If $n+1$ is a power of a prime number, then by [7] and [8], the monodromy group is maximal. Therefore, it suffices to check that the square $q(\alpha)$ is of the form $2 p-2-\frac{(2 g-\epsilon)^{2}}{2 n+2}$, with $p \geq g$.

The original proof was based on three ingredients: the 1st was a deformation theoretic statement, saying that rational curves whose deformations cover a divisor in irreducible holomorphic symplectic manifolds are non-obstructed [1, Corollary 3.5]. The 2nd is the characterization of polarized parallel transport operators on polarized irreducible holomorphic symplectic varieties $(X, H)$ of $K_{n}(A)$-type [9, Theorem 1.1] that allows to obtain an explicit description of the polarized deformation equivalence [9, Theorem 4.2]. These two ingredients are true. The 3rd argument consists in the construction of explicit examples of uniruled divisors on the generalized Kummer variety associated with a polarized abelian surface $\left(A, H_{A}\right)$ with $N S(A)=\mathbb{Z} H_{A}$ such that $p_{a}\left(H_{A}\right) \geq g \geq 2$. The construction is also correct, but the examples that we provided cannot yield all the possible primitive polarizations, as we tacitely and erroneously assumed in [9]. Even without taking the monodromy orbit into account, this is simply because it may happen that the number $2 p-2-\frac{(2 g-\epsilon)^{2}}{2 n+2}, \epsilon=0,1$ is positive even with $p<g$, which obviously renders our geometric argument empty. Indeed, the rational curves are constructed as $\mathfrak{g}_{n}^{1}$ on the normalization of a nodal curve of geometric genus $g$ lying in the hyperplane linear system $\left|H_{A}\right|$, which is supposed to have $p_{a}\left(H_{A}\right)=p$. We also take the occasion of this note to provide the full proof (Proposition 1.1) of a technical point that we claimed in [9, Section 4.2] to follow from a dimension count as 
in $[12$, Example 4.1, 3)]. The statement is correct, but the argument cannot be the same as in [12, Example 4.1, 3)] because we deal here with a locally closed subset (the Severi variety) of a complete linear system and not with the complete linear system.

The $K 3^{[n]}$-type case, initially treated in [1], is subject to the same considerations and will be treated in [2].

We realized our mistake after the appearance of [10], which provides counterexamples in the $K 3^{[n]}$-case that apply exactly in all cases not covered by the similar geometric constructions for the Hilbert scheme of points on a general projective $K 3$. Contrary to the $K 3^{[n]}$-type case as far as we know there are no known counterexamples to the existence of uniruled divisors ruled by a primitive curve class in the $K_{n}(A)$-type case. Nevertheless, we have no reasons to believe that the $K_{n}(A)$-type case could be exempt from this type of sporadic pathologies.

\section{Existence of Uniruled Divisors on $K_{n}(A)$}

In [9, Section 4.2 "Examples"] we claimed that "the natural map from $\widetilde{\mathscr{C}}_{g+1}^{1} \rightarrow A^{[g+1]}$ is finite onto its image" invoking a dimension count made in [12, Example 4.1, 3)]. However, the same argument cannot work because we do not work with the full continuous system but with a locally closed subset (the Severi variety). Hence, we take the occasion to provide a full proof of that statement in the following.

Proposition 1.1. Let $g$ be an integer $\geq 2$ and $\left(A, H_{A}\right)$ be a general polarized abelian surface with $p_{a}\left(H_{A}\right)=: p \geq g$. Then $A^{[g+1]}$ contains a uniruled divisor covered by the $\mathfrak{g}_{g+1}^{1}$ on nodal genus $g$ curves in the continuous system $\left\{H_{A}\right\}$.

Proof. To prove the statement we can actually work over a very general polarized abelian surface, so let us suppose that $N S(A)=\mathbb{Z} H_{A}$. We will prove this statement by induction on $g$. It is sufficient to show it on the symmetric product of $A$.

Observe that, by [4, Theorem. 1.1], for all $2 \leq g \leq p_{a}\left(H_{A}\right)$, that the Severi variety parametrizing nodal genus $g$ curves inside $\left\{H_{A}\right\}$ is non-empty of the expected dimension $g$.

It is sufficient to show the claim on the symmetric product $A^{(g+1)}$ of $A$. More precisely, we will prove the following statement: there exists an irreducible component $V$ of the (Zariski closure of the) Severi variety parametrizing nodal genus $g$ curves inside $\left\{H_{A}\right\}$ such that, if $\mathscr{C}_{V} \rightarrow V$ denotes the universal curve and $\mathscr{C}_{V}^{(g+1)} \rightarrow V$ the relative symmetric product, the natural morphism

$$
\mathscr{C}_{V}^{(g+1)} \rightarrow A^{(g+1)}
$$


is generically finite onto its image. Note that this is equivalent to saying that $(g+1)$ generic points on a generic curve of the family lie only on a finite number of curves of the family.

\section{Indeed as}

$$
\operatorname{dim} \mathscr{C}_{V}^{(g+1)}=\operatorname{reldim}\left(\mathscr{C}_{V}^{(g+1)}\right)+\operatorname{dim} V=(g+1)+g=2 g+1
$$

it follows that the image is a divisor inside $A^{(g+1)}$. Since the $k$-th symmetric product of a curve is uniruled for $k$ greater than the genus of the curve, as a by-product we have that such divisor is uniruled.

Note also that positive dimensional fibers of the morphism $\mathscr{C}_{V}^{(g+1)} \rightarrow A^{(g+1)}$ cannot lie in a fiber of $\mathscr{C}_{V}^{(g+1)} \rightarrow V$, as $C_{t}^{(g+1)}$ injects into $A^{(g+1)}$ for every $t \in V$.

We start with the case $g=2$. Let $C$ be one of the (finitely many) nodal curves of geometric genus 2 inside the linear system $\left|H_{A}\right|$. In this case the points of the component $V$ of the Severi variety containing $C$ are given by all the translates of $C$. The 3rd symmetric product $C^{(3)}$ injects as a three-dimensional subvariety inside $A^{(3)}$. The action of $A$ on $C^{(3)}$ by translation has no positive-dimensional stabilizer (as $A$ is general, hence simple). Therefore the orbit of $C^{(3)}$ under this action is a divisor. Using the notation above such divisor is the image of $\mathscr{C}_{V}^{(2+1)}$ in $A^{(3)}$.

By inductive hypothesis, there exists an irreducible component $W$ of the (Zariski closure of the) Severi variety parametrizing nodal genus $g-1$ curves inside $\left\{H_{A}\right\}$ such that, if $\mathscr{C}_{W} \rightarrow W$ denotes the universal curve and $\mathscr{C}_{W}^{(g)} \rightarrow W$ the relative symmetric product, the natural morphism

$$
\mathscr{C}_{W}^{(g)} \rightarrow A^{(g)}
$$

is generically finite onto its image.

Now let $V$ be (the Zariski closure of) an irreducible component of the Severi variety of nodal genus $g$ curves in $\left\{H_{A}\right\}$ obtained by smoothing one node of the curves in $W$ (which can be done by the regularity of the Severi variety, [3, Example 1.3]). By construction $W \subset V$. Let $\mathscr{C}_{V} \rightarrow V$ be the universal curve. Its restriction over $W$ yields a map $\mathscr{C}_{W} \rightarrow W$. Let $D$ be the image of the morphism

$$
\mathscr{C}_{V}^{(g+1)} \rightarrow A^{(g+1)}
$$

Observe that $D$ contains the image $D_{W}$ of

$$
\mathscr{C}_{W}^{(g+1)} \rightarrow A^{(g+1)}
$$


We claim that by the inductive hypothesis $D_{W}$ has codimension 2, or, equivalenty, that the morphism $\mathscr{C}_{W}^{(g+1)} \rightarrow A^{(g+1)}$ is generically finite onto its image. Indeed if $\xi=x_{1}+$ $\ldots+x_{g+1}$ is a generic point of the image, then, say, $x_{1}+\ldots+x_{g}$ is a generic point of the image of the morphism $\mathscr{C}_{W}^{(g)} \rightarrow A^{(g)}$. By the inductive hypothesis the points $x_{1}, \ldots, x_{g}$ lie on finitely many curves of the family $W$, a fortiori that will be true for $x_{1}, \ldots, x_{g}, x_{g+1}$ and the claim follows.

We want to prove that $D$ contains $D_{W}$ strictly. If this were not the case, by irreducibility, we would have $D=D_{W}$. Let $U \subset D$ be an open subset over which the morphisms $\mathscr{C}_{W}^{(g+1)} \rightarrow A^{(g+1)}$ and $\mathscr{C}_{V}^{(g+1)} \rightarrow A^{(g+1)}$ are smooth, and let $p_{1}+p_{2}+\cdots+p_{g+1}$ be a point in $U$. Let $C$ be a nodal genus $g$ curve in $V$ containing these points. Let us fix the 1 st $g$ points $p_{1}, \ldots, p_{g}$. By induction these points are contained inside a finite number of curves of genus $g-1$ belonging to $W$. Let $B_{1}, \ldots, B_{m}$ be all such curves. Let $U_{C} \subset C$ be an open subset such that for all $q \in U_{C}$ we have $p_{1}+\ldots+p_{g}+q \in U$. As we have seen above $p_{1}, \ldots, p_{g}, q$ lie on finitely many curves of genus $g-1$ belonging to $W$, and these curves must be $B_{1}, \ldots, B_{m}$. Therefore, as $q$ varies in $U_{C}$, we deduce that $U_{C}$ is a subset of a finite union of genus $(g-1)$ curves. As $C$ is irreducible, there is an $i$ such that $C=B_{i}$, which is clearly a contradiction. Therefore, $D$ must strictly contain $D_{W}$ and be a divisor, which is necessarily uniruled.

The rest of the proof remains the same and we refer the reader to [9] for the details.

\section{Where It Does Not Work}

In this section we prove that, for every dimension, there is at most a finite number of components of the moduli space of polarized manifolds $(X, H)$ of $K_{n}(A)$-type where the strategy of the previous section does not work. The uniruled divisors we constructed have a cohomology class that is a multiple of $H_{A}-(2 g) \tau$ (or $H_{A}-(2 g-1) \tau$ ) where $2 p-2=$ $H_{A}^{2}$ and $H_{A}$ is the primitive polarization on the abelian surface. We have the following:

Proposition 2.1. $\quad$ Let $X$ be a projective irreducible holomorphic symplectic variety of $K_{n}(A)$-type. Let $C \in H_{2}(X, \mathbb{Z}) \cap N_{1}(X)$ be a primitive class such that its square $q(C)$ with respect to the Beauville-Bogomolov form is $>n+1$. Then, the class $C$ is deformation equivalent to the class of one of the curves constructed in the previous section.

Proof. We know by [9, Theorem 4.2] that $C$ is deformation equivalent to either $H_{A}-2 g \tau$ or $H_{A}-(2 g-1) \tau$, with $g \leq n+1$. If $q(C)>n+1$, the square of $H_{A}-(2 n+2) \tau$ is positive, 
that is, $H_{A}^{2}=2 p-2$ with $p>n+1$. Thus, $p>n+1 \geq g$, which means that $H_{A}-2 g \tau$ can be represented by the class of a $\mathfrak{g}_{g+1}^{1}$ on a nodal curve in $\left\{H_{A}\right\}$.

Corollary 2.2. Let $\mathscr{M}_{n}$ be the moduli space of all polarized manifolds of $K_{n}(A)$-type with $n$ fixed. Then, the number of components of $\mathscr{M}_{n}$ whose general points $(X, H)$ do not have a uniruled divisor ruled by a rational curve of primitive class is at most finite.

Proof. The components of $\mathscr{M}_{n}$ are in bijective correspondence with the monodromy orbits of a given class of positive square in $L_{n}:=U^{3} \oplus(-2 n-2) \cong H^{2}(X, \mathbb{Z})$; see $[11$, Theorem 2.8].

For a fixed square of $H$, there is a finite number of orbits (computed again in [11, Theorem 2.8]), so it follows that if $X$ has a uniruled divisor when $q(H)$ is big enough, our claim will hold. The dual curve to $H$ is given by $H / \operatorname{div}(H)$, where $\operatorname{div}(H)$ is the divisibility of $H$, which is the positive generator of the ideal $q\left(H, H^{2}(X, \mathbb{Z})\right)$. The divisibility is at most $2 n+2$; therefore, if $q(H) \geq(2 n+2)^{2}(n+1)$ the dual curve has square at least $n+1$, so that Proposition 2.1 applies and our claim follows.

Corollary 2.3. Let $X$ be a projective manifold of $K_{n}(A)$-type with Picard rank at least two. Then $X$ has an ample divisor ruled by primitive rational curves.

Proof. Since $X$ is projective and has Picard rank at least two, its Picard lattice is indefinite and contains primitive elements of positive arbitrary Beauville-Bogomolov square and so does the ample cone. Let $H$ be an ample divisor such that $q(H) \geq(2 n+$ $2)^{2}(n+1)$. Let $C$ be its dual curve in $H_{2}(X, \mathbb{Z})$. As the divisibility of $H$ is at most $2 n+2$ it follows that $q(C) \geq n+1$, and Proposition 2.1 yields our claim.

Remark 2.4 The estimate of Proposition 2.1 is definitely not sharp; indeed, all primitive curves of positive square on manifolds of $K_{n}(A)$-type with $n \leq 5$ are deformation equivalent to the curves we construct in Proposition 1.1. Indeed, by [9, Theorem 4.2] we can suppose that our pair $(X, C)$ with $q(C)>0$ is $\left(K_{n}(A), H_{A}-\mu \tau\right)$ with $0 \leq \mu \leq n+1$ and $A$ is an abelian surface of genus $p$. The class $H_{A}-\mu \tau$ is given by the class of the rational curves constructed in Proposition 1.1, which have class $H_{A}-2 g \tau$, with the eventual addition of a tail of class $\tau$, so that $2 g \leq n+2$. By contradiction let us suppose that $g>p$ and $n \leq 5$. We have $q\left(H_{A}-2 g \tau\right)=2 p-2-2 \frac{g^{2}}{n+1} \leq 2 p-2-2 \frac{(p+1)^{2}}{n+1} \leq$ $2 p-2-2 \frac{(p+1)^{2}}{6}$. However, the last value is never positive; hence, $q\left(H_{A}-2 g \tau\right)$ cannot be positive and we reach a contradiction. Analogously, for $C=H_{A}-(2 g-1) \tau$, we have $q(C) \leq \frac{20 p-25-4 p^{2}}{12}$ with $g \geq p+1$ and $2 g \leq n+2$, which is again not positive. 


\section{Funding}

G.M. is supported by "Progetto di ricerca INdAM per giovani ricercatori: Pursuit of IHS". G.P. is partially supported by the Project ANR-16-CE40-0008 "Foliage".

\section{Acknowledgments}

We would like to thank Q. Yin for useful discussions and F. Charles for reading a preliminary version. G.M. would like to thank the Université de Lorraine for hosting him while this work was finished.

\section{References}

[1] Charles, F. and G. Pacienza. "Families of rational curves on holomorphic symplectic varieties." Preprint arXiv:1401.4071v2 [math.AG].

[2] Charles, F., G. Mongardi, and G. Pacienza. "Families of rational curves on holomorphic symplectic varieties of $K 3^{[n]}$-type." (forthcoming).

[3] Chiantini, L. and E. Sernesi. "Nodal curves on surfaces of general type." Math. Ann. 307 (1997): 41-56.

[4] Knutsen, A. L., M. Lelli-Chiesa, and G. Mongardi. "Severi varieties and Brill-Noether theory of curves on abelian surfaces." J. Reine Angew. Math. no. 749 (2019): 161-200.

[5] Knutsen, A.-L., M. Lelli-Chiesa, and G. Mongardi. "Wall divisors and algebraically coisotropic subvarieties of irreducible holomorphic symplectic manifolds." Trans. Amer. Math. Soc. 371, no. 2 (2019): 1403-38.

[6] Lange, H. and E. Sernesi. "Severi varieties and branch curves of abelian surfaces of type (1,3)." Int. J. Math. 13 (2002): 227-44.

[7] Markman, E. "The monodromy of generalized Kummer varieties and algebraic cycles on their intermediate Jacobians." (1805): 11574.

[8] Mongardi, G. "On the monodromy of irreducible symplectic manifolds." Algebr. Geom. 3 (2016): 385-91.

[9] Mongardi, G. and G. Pacienza. "Polarized parallel transport and uniruled divisors on deformations of generalized Kummer varieties." Int. Math. Res. Not. IMRN 2018, no. 11 (2018): 3606-20.

[10] Oberdieck, G., J. Shen, and Q. Yin. "Rational curves in the Fano varieties of cubic 4-folds and Gromov-Witten invariants." Preprint arXiv:1805.07001v1.

[11] Onorati, C. "Connected components of moduli spaces of generalized Kummer varieties." Preprint arXiv:1608.06465.

[12] Voisin, C. "Remarks and Questions on Coisotropic Subvarieties and 0-cycles of Hyper-Kähler Varieties." In K3 Surfaces and Their Moduli. Progress in Mathematics. Birkhäuser: Cham, Proceedings of the Schiermonnikoog Conference 2014. 